\title{
Abundances of disk and bulge giants from high-resolution optical spectra
}

\section{II. $\mathrm{O}, \mathrm{Mg}, \mathrm{Ca}$, and $\mathrm{Ti}$ in the bulge sample ${ }^{\star}$ (Corrigendum)}

\author{
H. Jönsson ${ }^{1,2,3}$, N. Ryde ${ }^{1}$, M. Schultheis ${ }^{4}$, and M. Zoccali ${ }^{5,6}$ \\ ${ }^{1}$ Lund Observatory, Department of Astronomy and Theoretical Physics, Lund University, Box 43, 22100 Lund, Sweden \\ e-mail: henrikj@astro.lu.se \\ 2 Instituto de Astrofísica de Canarias (IAC), 38205 La Laguna, Tenerife, Spain \\ 3 Universidad de La Laguna, Dpto. Astrofísica, 38206 La Laguna, Tenerife, Spain \\ ${ }^{4}$ Observatoire de la Côte d'Azur, Boulevard de l'Observatoire, BP 4229, 06304 Nice Cedex 4, France \\ 5 Instituto de Astrofísica, Pontificia Universidad Católica de Chile, Av. Vicuña Mackenna 4860, $782-0436$ Macul, Santiago, Chile \\ ${ }^{6}$ Millennium Institute of Astrophysics, Av. Vicuña Mackenna 4860, 782-0436 Macul, Santiago, Chile
}

A\&A 598, A101 (2017), DOI: 10.1051/0004-6361/201629129

Key words. Galaxy: bulge - Galaxy: evolution - stars: abundances - errata, addenda

An error occurred during the production process. The subtitle of the article in the published version was "II. O, Mg, Co, and Ti in the bulge sample". Here, we correct this to "II. O, $\mathrm{Mg}, \mathrm{Ca}$, and Ti in the bulge sample".

\footnotetext{
* Based on observations collected at the European Southern Observatory, Chile (ESO programs 71.B-0617(A), 073.B-0074(A), and 085.B-0552(A)).
} 